\title{
Robust adaptive controller for wheel mobile robot with disturbances and wheel slips
}

\author{
Nga Thi-Thuy Vu, Loc Xuan Ong, Nam Hai Trinh, Sen Thi Huong Pham
}

Hanoi University of Science and Technology, Viet Nam

\begin{tabular}{l} 
Article Info \\
\hline Article history: \\
Received Jun 7, 2020 \\
Revised Jun 23, 2020 \\
Accepted Aug 5, 2020 \\
\hline Keywords: \\
Adaptive controller \\
Disturbance observer \\
Stability analysis \\
Wheel mobile robot \\
Wheel slips
\end{tabular}

Article Info

Received Jun 7, 2020

Revised Jun 23, 2020

Accepted Aug 5, 2020

\section{Keywords:}

Disturbance observer

Stability analysis

Wheel slips

\begin{abstract}
In this paper an observer based adaptive control algorithm is built for wheel mobile robot (WMR) with considering the system uncertainties, input disturbances, and wheel slips. Firstly, the model of the kinematic and dynamic loops is shown with presence of the disturbances and system uncertainties. Next, the adaptive controller for nonlinear mismatched disturbance systems based on the disturbances observer is presented in detail. The controller includes two parts, the first one is for the stability purpose and the later is for the disturbances compensation. After that this control scheme is applied for both two loops of the system. In this paper, the stability of the closed system which consists of two control loops and the convergence of the observers is mathematically analysed based on the Lyapunov theory. Moreover, the proposed model does not require the complex calculation so it is easy for the implementation. Finally, the simulation model is built for presented method and the existed one to verify the correctness and the effectiveness of the proposed scheme. The simulation results show that the introduced controller gives the good performances even that the desired trajectory is complicated and the working condition is hard.
\end{abstract}

This is an open access article under the $\underline{C C B Y-S A}$ license.

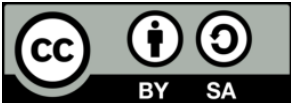

\section{Corresponding Author:}

Nga Thi-Thuy Vu,

School of Electrical Engineering,

Hanoi University of Science and Technology,

Hanoi, Viet Nam.

Email: nga.vuthithuy@ hust.edu.vn

\section{INTRODUCTION}

Nowadays, wheeled mobile robots (WMR) are widely used in many fields of the life. Mobile robots are self-moving vehicles and are versatile with many indoor and outdoor applications [1]. Therefore, problems of wheeled mobile robot are the interesting topics in both scientific researches and practical applications. However, the control of WMR is not easy because the WMR is an underactuated system. Moreover, the working environment of the WMR is changed depending on the applications. Different working environments of the WMR will have different types of wheel slip depending on many factors, such as an unknown centrifugal force possibly acting on the WMR when it moves in a circular path, an external force acting on the WMR, or a weak frictional force between the slippery floor and the wheels. So the controllers with the assumption of "pure rolling without slip" such as the sliding mode control [2-4], the adaptive control [5-9] or the back stepping control [10] cannot satisfy the requirements on the stability as well as the tracking of the system.

In recent years, to solve the problem of disturbances, some methods have been proposed to control the WMR. In [11], the tracking controller is cooperated with a disturbances observer and an adaptive compensator to deal with the system uncertainties. By this, the tracking error and the adaptive gain are 
bounded. In [12], the $\mu$-synthesis method based robust trajectory tracking control is used to deal with the problems of friction disturbances at low frequencies and modelling errors at high frequencies. The trajectory tracking controller is used in the outer loop to handle the nonholonomic constraints and a $\mu$-synthesis based robust control algorithm is designed for the inner loop to handle the friction disturbances and the modeling uncertainties with different frequencies. Due to careful consideration the effects of disturbances at low frequencies and the uncertainty of high frequencies, the trajectory tracking performance is significantly increased. However, the stability of the closed loop is not mentioned in this work. A robust controller based on disturbance observer for trajectory tracking of wheeled mobile robots presented in [13] is used to track the trajectory in the presence of disturbance and unknown parameters. In [14], the trajectory tracking problem for WMR under conditions of system uncertainties and external disturbances is addressed. By using a fuzzy logic system (FLS) to approximate the model and an adaptive fuzzy observer to estimate the unmeasured velocities, the position errors are minimized and the response of the system is fast. The FLSs are also used for the WMR systems in others researches [15-18].

The problems of wheel slips are also considered by many researchers. To eliminate the complex problems in the traditional backstepping design, a controller based on robust dynamic surface control (DSC) is built for the WMR in [19] with presence of skidding and slipping. Using the DSC technique and the nonlinear damping method, the effect of the uncertainties and disturbances is reduced significantly. The neural network adaptive controller based on the Reinforcement Learning method is shown in [20] to track the desired trajectory for the WMR with slipping and skidding. In [21], the particle swarm optimization (PSO) technique is used to tune the gain of the Lyapunov based controller to eliminate the effect of the slips. However, the reasonability of the scheme is not proven mathematically. In [22], a control scheme based on sliding mode control and a backstepping observer is built for the WMR with unknown slipping and model uncertainties. The algorithm cancels the influences of the system uncertainties, the disturbances, and the chattering of the sliding mode control. However, the illustrated results are so poor. A control technique which uses the fuzzy PID controller combining with the current sensor and the IUM sensor is introduced in [23] to reduce the effects of slipping caused by environmental factors. The results show that the controller scheme has the good effectiveness, but the stability of the system is not mentioned. A disturbance observer based robust controller with slipping and skidding considering is shown in [24]. However, the tracking error in this algorithm is so high.

In this paper, an adaptive algorithm based on the disturbance observer is proposed for the WRM with considering system uncertainties, external disturbances, and wheel slips. The scheme includes two control loops, the velocity control loop and the position control loop. The uncertainties and disturbances of each loop is observed and fed to the tracking controller. The contribution of the presented scheme is conclused as the following:

- Cancel the effect of the wheel slips and input disturbances on the operation of the sysem by the observers and adaptive controllers.

- The stability of each loops as well as overall system is mathematically proven. It is noted that proving the stability of the observer-based cascade control scheme which includes more than one observer and one controller is much more difficult than the single loop system.

- In comparision with the works in the same field, the proposed controller is quite simple, so it is easy to execute.

- The simulation shows good results under some harsh conditions (i.e., complicated trajectory, input disturbances, and wheel slips).

\section{ADAPTIVE CONTROLLER DESIGN FOR NONLINEAR MISMATCHED DISTURBANCES}

\subsection{System modelling}

Consider the WMR system which includes the wheel slips phenomena as shown in the Figure 1.

In the Figure 1, the meaning of the factors is as the following:

$\mathrm{G}\left(x_{G}, y_{G}\right) \quad$ the mass center of the platform

$\mathrm{M}\left(x_{M}, y_{M}\right) \quad$ the midpoint of the wheel shaft

$\mathrm{D}\left(x_{D}, y_{D}\right) \quad$ the desired point

$\mathrm{F}_{1}, \mathrm{~F}_{2} \quad$ the total longitudinal friction forces at the right and left wheel, respectively

$\mathrm{F}_{3} \quad$ the total lateral friction force along the wheel shaft

$\mathrm{F}_{4}, \mu \quad$ external force and moment acting on $\mathrm{G}$, respectively

$\gamma_{\mathrm{L}}, \gamma_{\mathrm{R}} \quad$ longitudinal slip factors of the left and right driving wheels, respectively

$\eta \quad$ the lateral slip factor along the wheel shaft

a the distance between the $\mathrm{M}$ and $\mathrm{G}$

$b \quad$ the haft of the wheel shaft 


\section{$\theta \quad$ the orientation of the WMR \\ $r \quad$ the radius of each wheel \\ $e_{p 1}, e_{p 2} \quad$ position errors between the points M and D in the MXY coordinate}

The kinematics and dynamics model of the WMR with the presence of wheel slips corresponding with coordinate illustrated in the Figure 1 are as the following [25]:

$$
\begin{aligned}
& \left\{\begin{array}{l}
\dot{x}_{M}=\vartheta \cos \theta-\dot{\eta} \sin \theta \\
\dot{y}_{M}=\vartheta \sin \theta+\dot{\eta} \cos \theta \\
\dot{\theta}=\omega
\end{array}\right. \\
& M \dot{v}+B v+Q \ddot{\gamma}+C \dot{\eta}+G \ddot{\eta}+\tau_{d}=\tau
\end{aligned}
$$

where $\vartheta$ is the forward velocity and $\omega$ is the angular velocity of the wheel mobile robot at point M. $\vartheta$ and $\omega$ are calculated as:

$$
\vartheta=\frac{r\left(\dot{\phi}_{R}+\dot{\phi}_{L}\right)}{2}+\frac{\dot{\gamma}_{R}+\dot{\gamma}_{L}}{2}, \quad \omega=\frac{r\left(\dot{\phi}_{R}-\dot{\phi}_{L}\right)}{2 b}+\frac{\dot{\gamma}_{R}-\dot{\gamma}_{L}}{2 b}
$$

in which $\dot{\phi}_{L}$ and $\dot{\phi}_{R}$ are the angular velocities of the left and right wheel, respectively

$$
\begin{aligned}
& v=\left[\begin{array}{ll}
\dot{\phi}_{R} & \dot{\phi}_{L}
\end{array}\right]^{T}, \quad \gamma=\left[\begin{array}{ll}
\gamma_{R} & \gamma_{L}
\end{array}\right]^{T}, M=\left[\begin{array}{ll}
m_{11} & m_{12} \\
m_{21} & m_{22}
\end{array}\right], m_{22}=m_{11}, m_{21}=m_{12} \\
& m_{11}=m_{G}\left(\frac{r^{2}}{4}+\frac{a r^{2}}{4 b^{2}}\right)+\frac{r^{2}}{4 b^{2}}\left(I_{G}+2 I_{D}\right)+2 m_{w} r^{2}+I_{w}, m_{12}=m_{G}\left(\frac{r^{2}}{4}-\frac{a^{2} r^{2}}{4 b^{2}}\right)-\frac{r^{2}}{4 b^{2}}\left(I_{G}+2 I_{D}\right) \\
& B=m_{G} \frac{r^{2}}{2 b} \omega\left[\begin{array}{cc}
0 & 1 \\
-1 & 0
\end{array}\right], Q=\left[\begin{array}{ll}
Q_{1} & Q_{2} \\
Q_{2} & Q_{1}
\end{array}\right], \quad Q_{1,2}=m_{G} \frac{r}{4}\left(1 \pm \frac{a^{2}}{b^{2}}\right) \pm \frac{r}{4 b}\left(I_{G}+2 I_{D}\right) \\
& C=m_{G} \frac{r}{2} \omega\left[\begin{array}{l}
1 \\
1
\end{array}\right], \quad G=m_{G} \frac{a r}{2 b}\left[\begin{array}{c}
1 \\
-1
\end{array}\right]
\end{aligned}
$$

$m_{G}$ and $m_{W}$ are the weight of the platform and each driving wheel, respectively, $I_{G}$ is the inertial moment of the platform about the vertical axis through point $G, I_{W}$ is the inertial moment of each wheel about its rotational axis, and $I_{D}$ is the inertial moment of each wheel about its diameter axis.
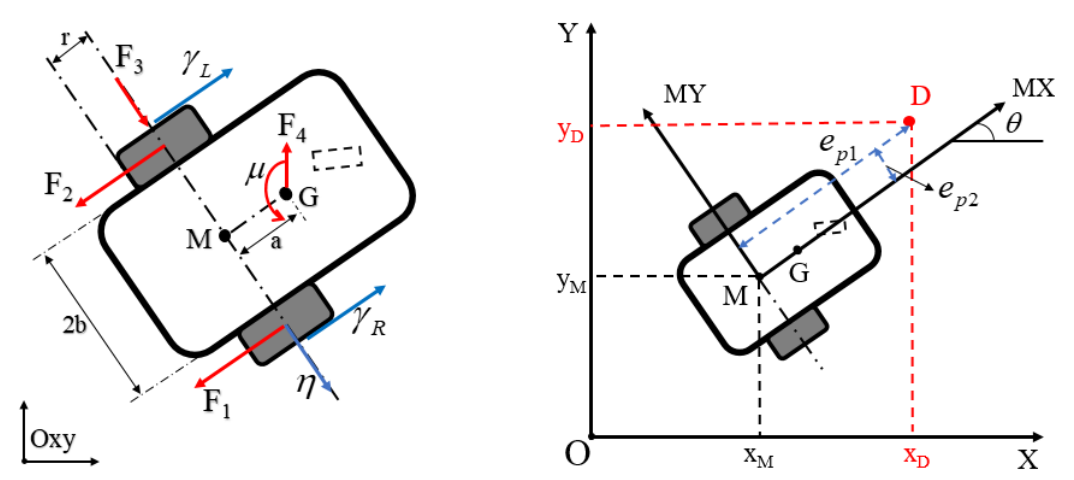

Figure 1. Block diagram of the WMR and the coordinate 


\subsection{Adaptive controller design for nonlinear mismatched disturbances}

Considering to the nonlinear system with mismatched disturbances as the following:

$$
\left\{\begin{array}{l}
\dot{x}=f(x)+g_{1}(x) u+d \\
y=h(x)
\end{array}\right.
$$

where $x \in R^{n x I}, d \in R^{n x l}, u \in R^{n x I}$ and $y \in R^{n x l}$ are the state vector, the disturbances vector, the control input vector, and the output vector, respectively. $f(x), g_{1}(x)$, and $h(x)$ are smooth functions. the following:

Considering to the system (3), a simple scheme is used to estimate the disturbances $d$ as

$$
\left\{\begin{array}{l}
\hat{d}=z+p(x) \\
\dot{z}=-L(x) z-L(x)\left[p(x)+f(x)+g_{1}(x) u\right]
\end{array}\right.
$$

where $\hat{d}$ is the estimates of $d, z$ is the internal state of the observer, $p(x)$ is the nonlinear function vector which will be designed, $L(x)$ is the observer gain vector defined by:

$$
L(x)=\frac{\partial p(x)}{\partial x}
$$

It is proven that the estimation error $e_{d}=d-\hat{d}$ is globally stable if the function $p(x)$ is chosen such that

$$
\dot{e}_{d}+L(x) e_{d}=0
$$

From the estimated vector $\hat{d}$ obtained in (4), the controller for the system (3) is designed with the following form:

$$
u=u^{*}(x)+\bar{u}(x) \hat{d}
$$

where $u^{*}(x)$ is the feedback component under the condition that the system is not affected by uncertainties as well as disturbances, $\bar{u}(x)$ is the disturbances compensator.

Define the new state variable

$$
\bar{x}=\left(\begin{array}{c}
x \\
e_{d}
\end{array}\right)
$$

Assuming that $g_{1}(x)$ is non-singular then the disturbances compensator is selected as the following:

$$
\bar{u}(x)=-g_{1}(x)^{-1}
$$

The derivative of (8) along with time is calculated based on (3), (6), and (7) as the following:

$$
\dot{\bar{x}}=\left[\begin{array}{c}
f(x)+g_{1}(x) \alpha(x)+e_{d} \\
-L(x) e_{d}
\end{array}\right]
$$

The stability of the closed loop system (10) is guaranteed by the following theorem.

Theorem 1: Assume that there exists a component $u^{*}(x)$ which keeps stable for the system (3) without considering the disturbances, a vector $L(x)$ guaranteeing the asymptotically stable for the observer (4), and a function $\bar{u}(x)$ satisfying (9) then the closed loop system (10) will be stable by the controller (7).

Proof: Choosing the Lyapunov function 
$V\left(e, e_{d}\right)=\frac{1}{2} e^{T} e+\frac{1}{2} e_{d} e_{d}^{T}$

where $e=x-x_{d}$ is the tracking error, $x_{d}$ is the reference value of $x$.

The derivative of (11) along with time is as the following:

$\dot{V}\left(e, e_{d}\right)=e^{T} \dot{e}+e_{d}^{T} \dot{e}_{d}$

Because original system is asymptotically stable with the control component $u^{*}(x)$ then $e^{T} \dot{e}<0$. This leads to the following:

$$
\dot{V}\left(e, e_{d}\right)<e_{d}^{T} \dot{e}_{d}=-e_{d}^{T} L(x) e_{d}
$$

This implies that if (6) is satisfied then $\dot{V}\left(e, e_{d}\right)<0$ or the closed loop system (10) is asymptotically stable.

$u^{*}(x)$ component calculation

Without considering the disturbances, the system (3) is rewritten as the following:

$\left\{\begin{array}{l}\dot{x}=f(x)+g_{1}(x) u \\ y=h(x)\end{array}\right.$

If $g_{1}(x)$ is non-singular then the following controller will be used:

$$
u=u^{*}(x)=g_{1}^{-1}(x)\left[-f(x)+\dot{x}_{d}-C e\right]
$$

where $x_{d}$ is the reference value of $x, e=x-x_{d}$ is the tracking error, $C$ is the positive constant vector.

Substituting (14) into (13) then the following result is obtained:

$$
\dot{e}=-C e
$$

Because $C$ is positive then the closed loop is asymptotically stable.

\subsection{Adaptive tracking controller design for WMR}

The purpose of this paper is to propose an adaptive controller to control the wheel mobile robot, i.e. the point $\mathrm{M}$ in the Figure 1 tracks the reference trajectory under the conditions that the wheels of the WMR slip, the system parameters are uncertainties, and the system is affected by external disturbances. The block diagram of the overall system is illustrated in Figure 2. The system includes two control loops: velocity control loop (inner loop) and position control loop (outer loop). The detail calculation for each loop is presented as the following:

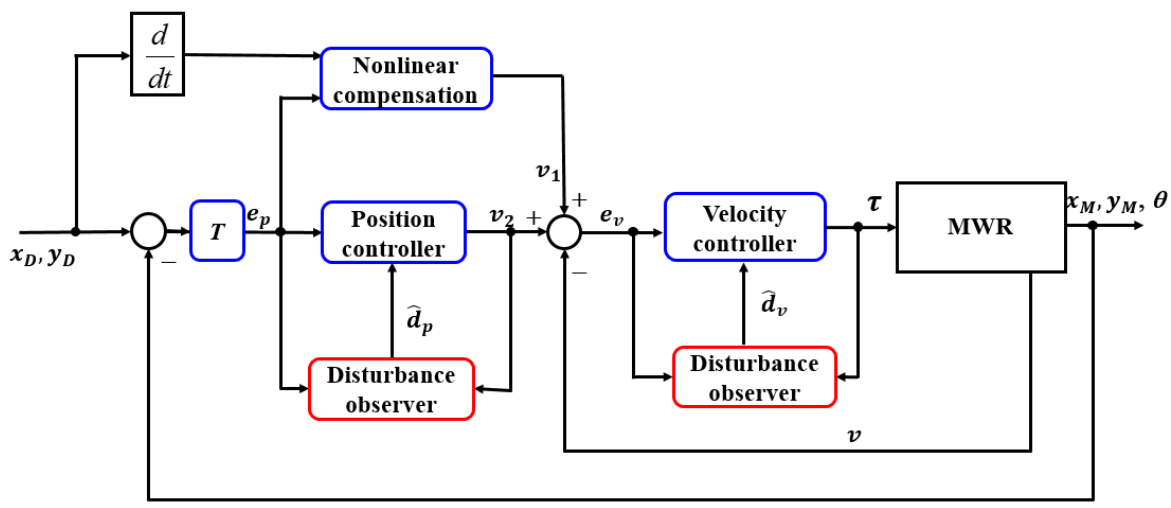

Figure 2. Block diagram of the proposed controller scheme 


\subsubsection{Dynamic controller design}

The dynamic model (2) is rewritten as the following form:

$$
\left.\dot{v}=-M^{-1} B v+M^{-1} \tau-M^{-1}\left(Q \ddot{\gamma}+C \dot{\eta}+G \ddot{\eta}+\tau_{d}\right)\right]
$$

Define $e_{v}=v-v_{d}$ where $v_{d}$ is the desired value of $v$. The error dynamic model obtained from (16) has the following formulation:

$$
\dot{e}_{v}=-M^{-1} B e_{v}+M^{-1} \tau-\dot{v}_{d}-M^{-1} B v_{d}-M^{-1}\left(Q \ddot{\gamma}+C \dot{\eta}+G \ddot{\eta}+\tau_{d}\right)
$$

or it can be shortened as:

$$
\dot{e}_{v}=-M^{-1} B e_{v}+M^{-1} \tau+d_{v}
$$

where $d_{v}=-\dot{v}_{d}-M^{-1} B v_{d}-M^{-1}\left(Q \ddot{\gamma}+C \dot{\eta}+G \ddot{\eta}+\tau_{d}\right)$ is the unknown component which need to be estimated.

The error dynamics model (18) has the form of (3), then the torque controller is obtained as the following:

$$
\tau=\left(B-M C_{1}\right) e_{v}-M \hat{d}_{v}
$$

Where

$$
\left\{\begin{array}{l}
\hat{d}_{v}=z_{v}+L_{1} e_{v} \\
\dot{z}_{v}=-L_{1} z_{v}-L_{1}\left(L_{1} e_{v}-M^{-1} B e_{v}+M^{-1} \tau\right)
\end{array}\right.
$$

in which $L_{l}$ and $C_{l}$ are positive scalars.

\subsubsection{Kinematic controller design} the follow:

In the MXY coordinate, the position error between the points $\mathrm{M}\left(\mathrm{x}_{\mathrm{M}}, \mathrm{y}_{\mathrm{M}}\right)$ and $\mathrm{D}\left(\mathrm{x}_{\mathrm{D}}, \mathrm{y}_{\mathrm{D}}\right)$ is as

$$
e_{p}=\left[\begin{array}{l}
e_{p 1} \\
e_{p 2}
\end{array}\right]=\left[\begin{array}{cc}
\cos \theta & \sin \theta \\
-\sin \theta & \cos \theta
\end{array}\right]\left[\begin{array}{l}
x_{D}-x_{M} \\
y_{D}-y_{M}
\end{array}\right]=T\left[\begin{array}{l}
x_{D}-x_{M} \\
y_{D}-y_{M}
\end{array}\right]
$$

where $T$ is the transformation matrix:

$$
T=\left[\begin{array}{cc}
\cos \theta & \sin \theta \\
-\sin \theta & \cos \theta
\end{array}\right]
$$

Derivative of (21) along with time under the condition of wheels slip, external disturbances is obtained as [19]:

$$
\dot{e}_{p}=\left[\begin{array}{c}
\dot{e}_{p 1} \\
\dot{e}_{p 2}
\end{array}\right]=\left[\begin{array}{cc}
\cos \theta & \sin \theta \\
-\sin \theta & \cos \theta
\end{array}\right]\left[\begin{array}{c}
\dot{x}_{D} \\
\dot{y}_{D}
\end{array}\right]+h v+d_{p}
$$

where

$$
h=\left[\begin{array}{cc}
\left(\frac{e_{p 2}}{b}-1\right) \frac{r}{2} & -\left(\frac{e_{p 2}}{b}+1\right) \frac{r}{2} \\
-\frac{e_{p 1} r}{2 b} & \frac{e_{p 1} r}{2 b}
\end{array}\right], d_{p}=\left[\begin{array}{c}
\left(\frac{\dot{\gamma}_{R}-\dot{\gamma}_{L}}{2 b}\right) e_{p 2}-\frac{\dot{\gamma}_{R}+\dot{\gamma}_{L}}{2} \\
-\left(\frac{\dot{\gamma}_{R}-\dot{\gamma}_{L}}{2 b}\right) e_{p 1}-\dot{\eta}
\end{array}\right]
$$


The controller $v$ is separated into two parts $v_{1}$ and $v_{2}$ where $v_{1}$ is the feedforward part using to compensate the nonlinear component in the model (22), $v_{2}$ is the feedback controller.

$$
\begin{aligned}
& v=v_{1}+v_{2} \\
& v_{1}=-h^{-1}\left[\begin{array}{cc}
\cos \theta & \sin \theta \\
-\sin \theta & \cos \theta
\end{array}\right]\left[\begin{array}{l}
\dot{x}_{D} \\
\dot{y}_{D}
\end{array}\right]
\end{aligned}
$$

Substituting (23) and (24) into (22) leads to:

$$
\dot{e}_{p}=h v_{2}+d_{p}
$$

Using control scheme proposed in the part 2, the controller $v_{2}$ has the following form:

$$
v_{2}=-h^{-1} C_{2} e_{p}+h^{-1} \hat{d}_{p}
$$

where

$$
\left\{\begin{array}{l}
\hat{d}_{p}=z_{p}+L_{2} e_{p} \\
\dot{z}_{p}=-L_{2} z_{p}-L_{2}\left(L_{2} e_{p}+h v_{2}\right)
\end{array}\right.
$$

in which $C_{2}$ and $L_{2}$ are positive scalars.

It is noted that when the WMR tracks the desired trajectory, the errors $e_{p 1}$ and $e_{p 2}$ go to zero. This leading to $\operatorname{det}(h)=-e_{p 1} \frac{r^{2}}{2 b} \rightarrow 0$ and $h$ is not invertable. To avoid this problem, the controller (26) is modified as the following:

$$
v_{2}= \begin{cases}-h^{-1} C_{2} e_{p}+h^{-1} \hat{d}_{p} & \text { if }\left|e_{p 1}\right| \geq \gamma \\ \frac{1}{\gamma}\left(C_{2} e_{p}+\hat{d}_{p}\right) & \text { if }\left|e_{p 1}\right|<\gamma\end{cases}
$$

where $\gamma$ is a small enough scalar.

\subsection{Overall stability}

The stability of the overall system which includes the WMR, the dynamic controller, the kinematic controller, and the observers is guaranteed by the following theorem.

Theorem 2: Assuming that the dynamics (16) with the controller (19) and the observer (20) is stable, also the kinematics (22) with the controller (23) and the observer (27) is stable. Then the position tracking error $e_{p}$ and the velocity error $e_{v}$ are asymptotically convergent to zero as time goes to infinity.

Proof: Choosing the Lyapunov function

$$
V=\frac{1}{2} e_{p}^{T} e_{p}+\frac{1}{2} e_{v}^{T} e_{v}+\frac{1}{2} e_{d p}^{T} e_{d p}+\frac{1}{2} e_{d v}^{T} e_{d v}
$$

where $e_{d p}=d_{p}-\hat{d}_{p}$ and $e_{d v}=d_{v}-\hat{d}_{v}$ are the estimation errors in the outer and inner loops, respectively.

The time derivative of (29) is obtained as the following:

$$
\dot{V}=e_{p}^{T} \dot{e}_{p}+e_{v}^{T} \dot{e}_{v}+e_{d p}^{T} \dot{e}_{d p}+e_{d v}^{T} \dot{e}_{d v}
$$

Using (6), (18), (19) (25) and (26), the (30) is rewritten as: 


$$
\begin{aligned}
\dot{V}= & e_{p}^{T}\left(-C_{2} e_{p}+e_{d p}\right)+e_{v}^{T}\left(-C_{1} e_{v}+e_{d v}\right)-e_{d p}^{T} L_{2} e_{d p}-e_{d v}^{T} L_{1} e_{d v} \\
= & -\left(e_{p}^{T} C_{2} e_{p}-e_{p}^{T} e_{d p}+e_{d p}^{T} L_{2} e_{d p}\right)-\left(e_{v}^{T} C_{1} e_{v}-e_{v}^{T} e_{d v}+e_{d v}^{T} L_{1} e_{d v}\right) \\
= & -\left(e_{p}^{T} e_{p}-e_{p}^{T} e_{d p}+e_{d p}^{T} e_{d p}\right)-\left(e_{v}^{T} e_{v}-e_{v}^{T} e_{d v}+e_{d v}^{T} e_{d v}\right) \\
& -\left(C_{2}-1\right) e_{p}^{T} e_{p}-\left(C_{1}-1\right) e_{v}^{T} e_{v}-\left(L_{1}-1\right) e_{d p}^{T} e_{d p}-\left(L_{2}-1\right) e_{d v}^{T} e_{d v}
\end{aligned}
$$

It is easy to see that $\dot{V} \leq 0$ or $e_{p}, e_{v}, e_{d p}$, and $e_{d v}$ are bounded if $C_{1}, C_{2}, L_{1}$, and $L_{2}$ are larger than one. Consider to the function:

$$
V_{1}=V-\int\left(\dot{V}+e_{p}^{T} e_{p}+e_{v}^{T} e_{v}\right) d t
$$

Its first and second order time derivative is given by (32) and (33), respectively, as the following:

$$
\begin{aligned}
& \dot{V}_{1}=-e_{p}^{T} e_{p}-e_{v}^{T} e_{v} \\
& \ddot{V}_{1}=-e_{p}^{T} \dot{e}_{p}-e_{v}^{T} \dot{e}_{v}=-e_{p}^{T}\left(-C_{2} e_{p}+e_{d p}\right)-e_{v}^{T}\left(-C_{1} e_{v}+e_{d v}\right)
\end{aligned}
$$

Since $e_{p}, e_{v}, e_{d p}$, and $e_{d v}$ are bounded then $\ddot{V}_{1}$ is bounded. Using Barbalat's lemma it is clearly to see that $e_{p}$ and $e_{v}$ asymptotically converge to zero as time goes to infinity.

\section{CONTROL STRATEGY VERIFICATION}

In order to verify the effectiveness of the proposed scheme, two simulations are executed by Matlab/Simulink for the heart shape and the oval shape. In both cases, it is assumed that the WMR operates under the effect of parameter uncertainties (20\% differences from nominal parameters), input disturbances $\tau_{d}=\left[\begin{array}{llll}1+\sin (0.2 t) & 1+\cos (0.2 t)\end{array} \quad(\mathrm{N} . \mathrm{m})\right.$, and the slips $\left[\begin{array}{lll}\dot{\gamma}_{R} & \dot{\gamma}_{L} & \dot{\eta}\end{array}\right]^{T}=\left[\begin{array}{llll}1+\sin t & 1+\cos t & \sin t\end{array}\right]^{T} \quad(\mathrm{~m} / \mathrm{sec})$. Also, the same simulation is done with the scheme which is presented in [18] for comparison.

The parameters of the WMR is as the following: $m_{G}=10 \mathrm{~kg}, m_{W}=2 \mathrm{~kg}, I_{G}=4 \mathrm{kgm}^{2}, I_{W}=0.1 \mathrm{kgm}^{2}$, $I_{D}=0.05 \mathrm{kgm}^{2}, a=0.2 \mathrm{~m}, b=0.3 \mathrm{~m}, r=0.15 \mathrm{~m}$. The control parameters are chosen as:

$$
C_{1}=C_{2}=2, L_{1}=200, L_{2}=300 \text {. }
$$

- Case 1: The reference trajectory is oval in shape with the equation:

$$
x_{D}=1+\sin t, \quad y_{D}=\cos t
$$

The initial positions of the robot are $\left[\begin{array}{lll}x & y & \theta\end{array}\right]=\left[\begin{array}{lll}0.7 & 1.2 & 0\end{array}\right]$. The simulation results are shown in Figures 3 and 4. Thoroughly, the Figure 3 illustrates the reference trajectory, the response of the proposed method, and the response of the sliding mode control method. It can be seen from the Figure 3 that, the tracking of the robot which is controlled by the proposed algorithm is better than the sliding mode one. This difference is reflected on Figure 4. In Figure 4, the position errors of the two controller schemes are shown in $x$-axis and $y$-axis. One more time, it is easy to see that the position error of the sliding mode control is quite large (about $0.11 \mathrm{~m}$ ) but in the case of the proposed controller this error is almost zero.

- Case 2: The reference trajectory is heart in shape with the equation:

$$
x_{D}=12 \sin \left(\frac{\pi}{20} t\right)-4 \sin \left(\frac{3 \pi}{20} \cdot t\right), \quad y_{D}=13 \cos \left(\frac{\pi}{20} t\right)-5 \cos \left(\frac{2 \pi}{20} t\right)-2 \cos \left(\frac{3 \pi}{20} t\right)-\cos \left(\frac{\pi}{20} t\right)
$$

In this case, the initial position of the robot is $\left[\begin{array}{lll}x & y & \theta\end{array}\right]=\left[\begin{array}{lll}-1.2 & 4 & 0\end{array}\right]$. The reference trajectory is much more complicated and the velocity is also more change. However, as indicated in Figures 5 and 6 , the proposed controller scheme still gives the good performances with small tracking error (almost zero) in both position and velocity loops. Meanwhile, the position error in the sliding mode control is about $0.1 \mathrm{~m}$. 
The response time of two schemes is similar, but the maximum oscillation amplitude of the SMC method at the initial time is double that of the proposed method $(0.4 \mathrm{~m}$ and $0.2 \mathrm{~m})$.

The numerical analysis for above results is depicted in the Table 1. From the above results it can be seen that the proposed observer based adaptive controller scheme give the good tracking. In comparison with the SMC, the presented algorithm gives the performances with smaller tracking error and oscillation, faster response even though the reference trajectory is complicated and the velocity is much change.

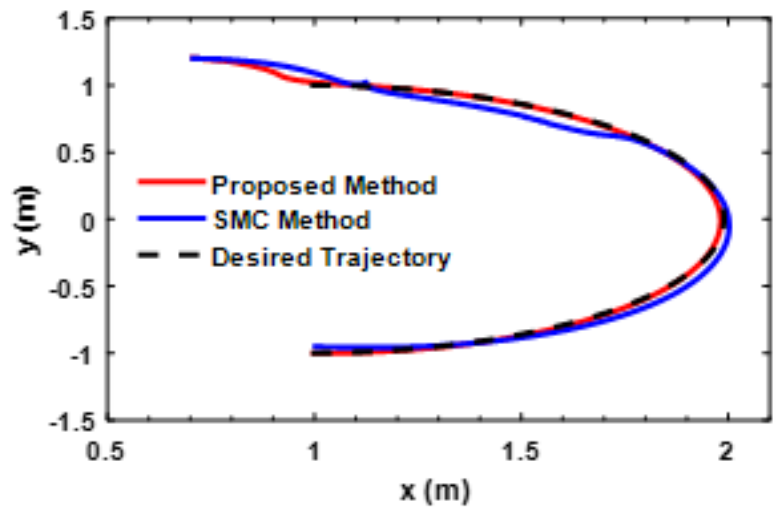

Figure 3. Trajectory tracking of the proposed controller and the sliding mode controller (SMC)

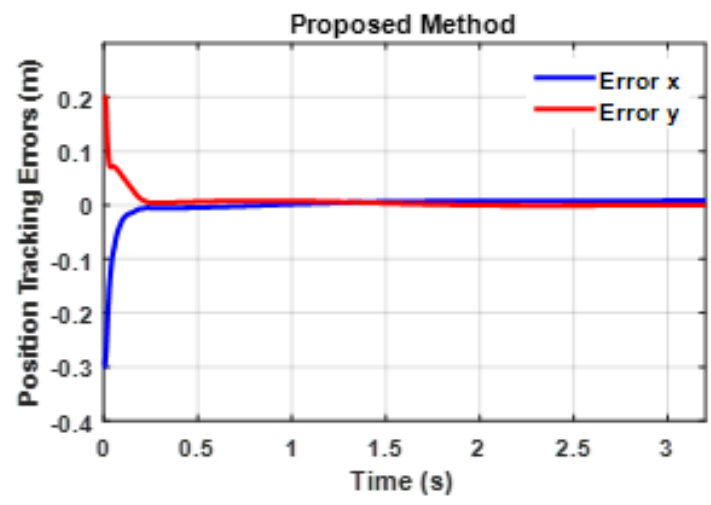

(a)

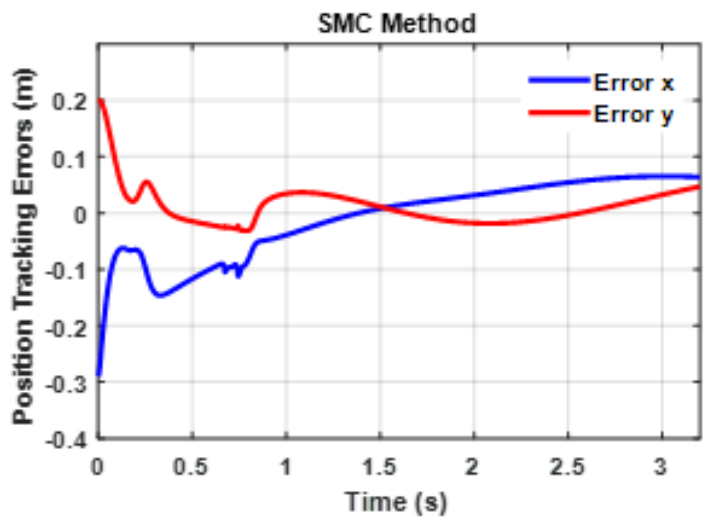

(b)

Figure 4. Tracking error of the proposed (a) and the sliding mode (b) controllers

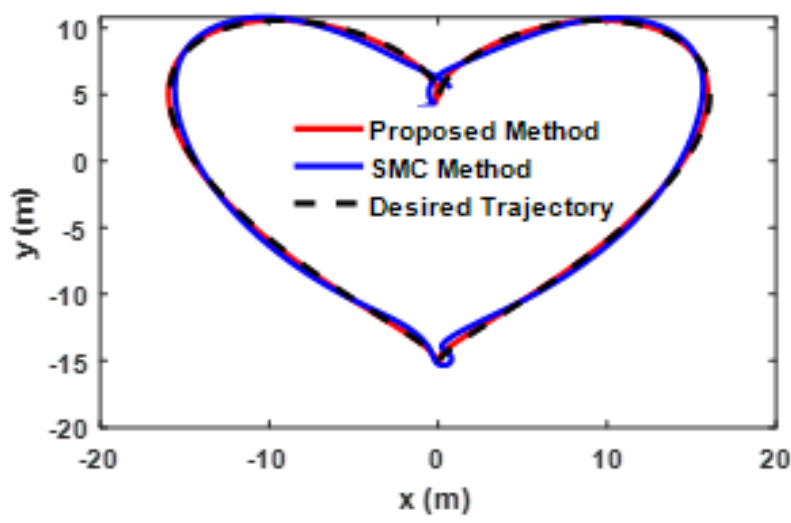

Figure 5. Trajectory tracking of the proposed controller and the SMC 

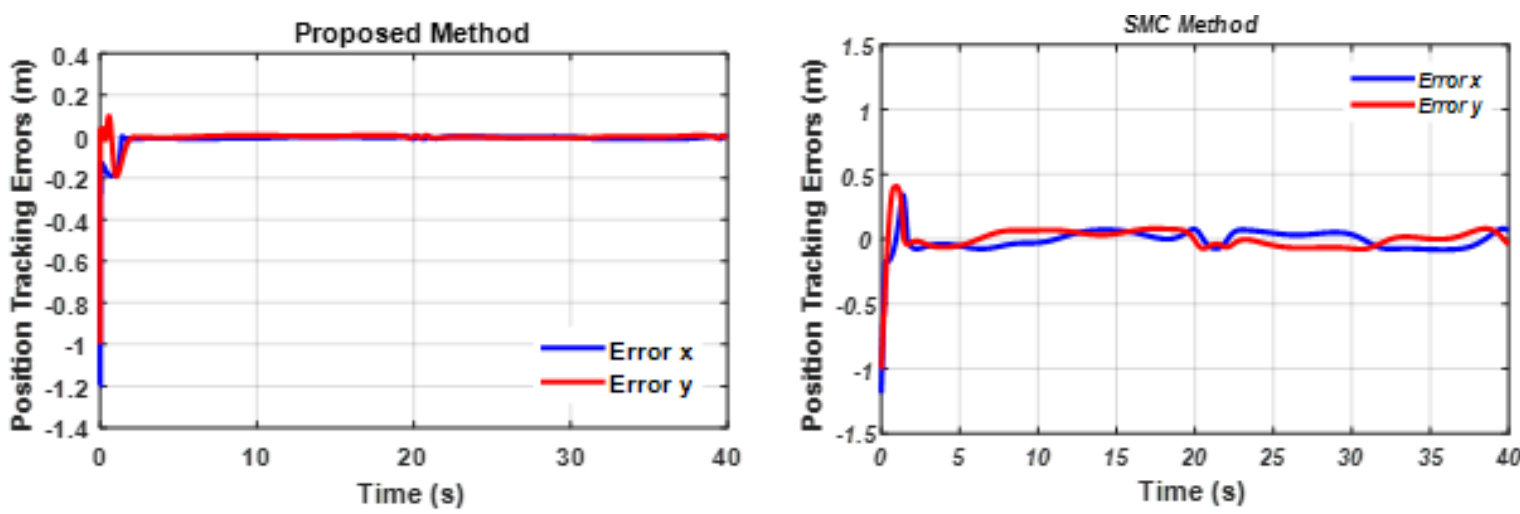

Figure 6. Tracking error of the proposed and the sliding mode controllers

Table 1. Performance index of the proposed and sliding mode controllers in two cases

\begin{tabular}{ccccccc}
\hline & \multicolumn{2}{c}{ Steady state error $(\mathrm{m})$} & \multicolumn{2}{c}{ Overshoot $(\%)$} & \multicolumn{2}{c}{ Oscillation } \\
\hline & Proposed & SMC & Proposed & SMC & Proposed & SMC \\
Oval shape & Almost zero & 0.05 & No & No & No & Yes \\
Heart shape & Almost zero & 0.1 & 10 & 40 & No & Yes \\
\hline
\end{tabular}

\section{CONCLUSION}

In this paper an adaptive controller based on the disturbances observer is presented to deal with the problems of disturbances and wheel slips in the wheel mobile robot. The control system is separated into two loops, dynamic loop and kinematic loop. For each loop, the unknown component is estimated through an observer. Then, this value is fed to the controller to compensate the effect of the disturbances. In this paper, the stability of the overall system which includes two control loops as well as the convergence of the observers are mathematically proven based on Lyapunov theory and Brabalat's lemma (It is noted that the stability of the observer based controller proven for one loop structure is more easier than the two loops one). Moreover, this scheme does not need the complex calculation so it is easy for implementation. The properness of the proposed algorithm is verified through two simulations. In each simulation, the performances of the presented adaptive controller are compared with the sliding mode controller. The simulation results show that the adaptive controller gives the better responses in both cases, i.e. smaller tracking error, smaller oscillation, and fast response even that the trajectory is complicated and the velocity is much change.

\section{REFERENCES}

[1] G. Mester, "Application of mobile robots," 7th international Conference on Food Science, 2006.

[2] S. A. Tchenderli-Baham, F. Hamerlain, and N. Saadia, "Adaptive sliding mode for the control of a wheeled mobile robot," 15th International Conference on Control, Automation and Systems, 2015.

[3] Z. Cao, Y. Niu, "Finite-time sliding mode control of Markovian jump systems subject to actuator nonlinearities and its application to wheeled mobile manipulator," Journal of the Franklin Institute, vol. 355, pp. 7865-7894, 2018.

[4] A. Filipescu, V. Minzu, B. Dumitrascu, A. Filipescu, E. Minca, "Trajectory-tracking and discrete-time slidingmode control of wheeled mobile robots," IEEE International Conference on Information and Automation, 2011.

[5] L. Ding, S. Li, Y.-J. Liu, H. Gao, C. Chen, Z. Deng, "Adaptive neural network-based tracking control for full-state constrained wheeled mobile robotic system," IEEE Transactions on Systems, Man, and Cybernetics: Systems, vol. 47, pp. 2410-2419, 2017.

[6] K. Shojaei, "Neural adaptive output feedback formation control of type (m, s) wheeled mobile robots," IET Control Theory \& Applications, vol. 11, pp. 504-515, 2017.

[7] L. Li, et al., "Adaptive trajectory tracking of nonholonomic mobile robots using vision-based position and velocity estimation," IEEE Transactions on Cybernetics, vol. 48, pp. 571-582, 2018.

[8] Z. Peng, S. Yang, G. Wen, A. Rahmani, Y. Yu, "Adaptive distributed formation control for multiple nonholonomic wheeled mobile robots," Neurocomputing, vol. 173, pp. 1485-1494, 2016.

[9] M. Begnini, D. W. Bertol, N. A. Martins, "A robust adaptive fuzzy variable structure tracking control for the wheeled mobile robot: Simulation and experimental results," Control Engineering Practice, vol. 64, pp. 27-43, 2017.

[10] G. Yanfeng, Z. Hua, Y. Yanhui, "Back-Stepping and Neural Network Control of a Mobile Robot for Curved Weld Seam Tracking," Procedia Engineering, vol. 15, pp. 38-44, 2011.

[11] L. Xin, Q. Wang, J. She, Y. Li, "Robust adaptive tracking control of wheeled mobile robot," Robotics and Autonomous Systems, vol. 78, pp. 36-48, 2016. 
[12] Z. Deng, et al., "Modeling and $\mu$-synthesis Based Robust Trajectory Tracking Control of a Wheeled Mobile Robot," IFAC Proceedings Volumes, vol. 47, pp. 7221-7226, 2014.

[13] D. Huang, J. Zhai, W. Ai, S. Fei, "Disturbance observer-based robust control for trajectory tracking of wheeled mobile robots," Neurocomputing, vol. 198, pp. 74-79, 2016.

[14] S. Peng, W. Shi, "Adaptive Fuzzy Output Feedback Control of a Nonholonomic Wheeled Mobile Robot," IEEE Access, vol. 6, pp. 43414-43424, 2018.

[15] H. Shi, M. Xu, K.S. Hwang, "A fuzzy adaptive approach to decoupled visual servoing for a wheeled mobile robot," IEEE Transactions on Fuzzy Systems, 2019.

[16] X. Lu, J. Fei, "Velocity tracking control of wheeled mobile robots by fuzzy adaptive iterative learning control," IEEE 2016 Chinese Control and Decision Conference, pp. 4242-4247.

[17] B. Sumantri, E.H. Binugroho, I.M. Putra, R. Rokhana, "Fuzzy-PID controller for an energy efficient personal vehicle: Two-wheel electric skateboard," International Journal of Electrical \& Computer Engineering, vol. 9, pp. 2088-8708, 2019.

[18] M. Begnini, D. W. Bertol, and N. A. Martins, "A robust adaptive fuzzy variable structure tracking control for the wheeled mobile robot: Simulation and experimental results," Control Engineering Practice, vol. 64, pp. 27-43, 2017.

[19] S.-H. Yu, C.-H, Hyun, H. S. Kang, "Robust dynamic surface tracking control for uncertain wheeled mobile robot with skidding and slipping," IEEE International Conference on Control and Robotics Engineering, 2016.

[20] S. Li, et al., "Adaptive neural network tracking control-based reinforcement learning for wheeled mobile robots with skidding and slipping," Neurocomputing, vol. 283, pp. 20-30, 2018.

[21] N. K, Goswami, P. K. Padhy, "Gain tuning of Lyapunov function based controller using PSO for mobile robot control," 11th International Conference on Industrial and Information Systems, 2016.

[22] Y. Jinhua, Y. Suzhen, J. Xiao, "Trajectory tracking control of wmr based on sliding mode disturbance observer with unknown skidding and slipping," 2nd International Conference on Cybernetics, Robotics and Control, 2017.

[23] D.-E. Kim, H.-N. Yoon, K.-S. Kim, M. S. Sreejith, J.-M. Lee, "Using current sensing method and fuzzy PID controller for slip phenomena estimation and compensation of mobile robot," 14th International Conference on Ubiquitous Robots and Ambient Intelligence, 2017.

[24] M. Chen, "Disturbance attenuation tracking control for wheeled mobile robots with skidding and slipping," IEEE Transactions on Industrial Electronics, vol. 64, pp. 3359-3368, 2017.

[25] T. Nguyen, L. Le, "Neural network-based adaptive tracking control for a nonholonomic wheeled mobile robot with unknown wheel slips, model uncertainties, and unknown bounded disturbances," Turkish Journal of Electrical Engineering \& Computer Sciences, vol. 26, pp. 378-392, 2018. 